\title{
Desapoio dos gestores e desinstitucionalização das Práticas Integrativas e Complementares no Sistema Único de Saúde
}

\author{
Lack of management and institutional support \\ for Integrative and Complementary Practices in \\ the Brazilian Unified National Health System
}

\author{
Falta de apoyo de los gestores y \\ desinstitucionalización de las Prácticas \\ Integradoras y Complementarias en el \\ Sistema Único de Salud
}

\author{
Nelson Filice de Barros 1 \\ Priscila Maria Stolses Bergamo Francisco 1 \\ Leandra Andréia de Sousa 2
}

doi: 10.1590/0102-311X00062320

O artigo de Barbosa et al. ${ }^{1}$, intitulado Oferta de Práticas Integrativas e Complementares em Saúde na Estratégia Saúde da Família no Brasil, publicado no fascículo de janeiro do volume 36 de CSP, apresentou e discutiu a convergência da oferta das Práticas Integrativas e Complementares (PIC) na Estratégia Saúde da Família (ESF) com base no olhar de gestores e profissionais de saúde. Os resultados apresentados no artigo mostram, em consonância com dados nacionais 2,3,4 e internacionais 5,6, a baixa institucionalização das PIC nos sistemas nacionais de saúde e as consequências deste desapoio.

$\mathrm{O}$ artigo evidencia, de um lado, o baixo reconhecimento da oferta das PIC na ESF pelos gestores, e de outro, o protagonismo e iniciativa de profissionais da ESF para a ampliação da oferta das PIC. Evidencia, também, que 74,35\% da oferta das PIC acontecem na ESF no Sistema Único de Saúde (SUS), corroborando os impactos da ESF na melhoria dos indicadores de acesso, qualidade dos serviços, eficiência no sistema, proteção financeira, impacto na saúde da população e equidade 7,8. O artigo mostra, ainda, a discrepância significativa entre profissionais da ESF e gestores de serviços sobre o uso de PIC na atenção básica. Além de destacar que os profissionais autofinanciam sua formação em PIC, com baixo apoio e reconhecimento dos gestores.

As evidências citadas se baseiam nos dados de 8,6\% dos municípios brasileiros, e são semelhantes aos achados de investigação concluída em 2010 9,10 nos 645 municípios do Estado de São Paulo sobre a oferta da medicina homeopática no SUS. Nesse estudo contemporâneo à publicação da Portaria no 971/2006, que criou a Política Nacional de Práticas Integrativas e Complementares (PNPIC) 11, observou-se que 47 municípios paulistas ofertavam homeopatia e que entre os gestores de 42 cidades, $26 \%$ conheciam a PNPIC, $31 \%$ a conheciam pouco e $41 \%$ desconheciam. Concluiu-se naquele momento que a PNPIC era desconhecida pelos gestores da saúde e que os maiores responsáveis pela implantação e manutenção da homeopatia no SUS dos municípios eram os médicos homeopatas, que autofinanciaram sua formação e contavam com pequeno apoio dos gestores.

$\mathrm{O}$ artigo de Barbosa et al. resulta da análise dos dados do Inquérito Nacional de Práticas Integrativas e Complementares em Saúde no SUS, que foi financiado pelo único edital do Ministério da Saúde específico para a pesquisa sobre PIC no SUS em 2013 (Edital MCTI/CNPq/MS-SCTIE-Decit no 07/2013). Custeados por esse mesmo edital desenvolvemos o projeto A Oferta de Racionalidades Médicas e Práticas Integrativas e Complementares na Atenção Primária à Saúde da Região Metropolitana de Campinas e, entre outras coisas, concluímos que a oferta das PIC nos serviços da atenção básica está diretamente rela-
1 Faculdade de Ciências Médicas, Universidade Estadual de Campinas, Campinas, Brasil. 2 Universidade Federal de São Carlos, São Carlos, Brasil.

\section{Correspondência}

N. F. Barros

Faculdade de Ciências Médicas, Universidade Estadual de Campinas. Rua Tessália Vieira de Camargo 126, Campinas, SP 13083-887, Brasil.

nelfel@uol.com.br 
cionada à crença religiosa, formação profissional e tempo de trabalho dos coordenadores nos serviços de saúde que gerenciam.

De acordo com os resultados apresentados na Tabela 1 é possível verificar que os profissionais da ESF têm maior apoio para a oferta das PIC quando os gestores trabalham no serviço que gerenciam há mais de 10 anos, têm crenças religiosas diferentes das evangélicas e têm formação profissional em Medicina ou Odontologia.

\section{Tabela 1}

Práticas Integrativas Complementares (PIC) nas unidades básicas de saúde (UBS), de acordo com as características sociodemográficas dos responsáveis pelos serviços. Região Metropolitana de Campinas, São Paulo, Brasil, 2016.

\begin{tabular}{|c|c|c|c|c|c|}
\hline \multirow[t]{2}{*}{ Variáveis sociodemográficas } & \multicolumn{2}{|c|}{ PIC } & \multicolumn{2}{|c|}{ Não PIC } & \multirow[t]{2}{*}{ Valor de $\mathrm{p}$} \\
\hline & $\mathbf{n}$ & $\%$ & $\mathbf{n}$ & $\%$ & \\
\hline Sexo & & & & & 0,455 \\
\hline Masculino & 12 & 57,1 & 9 & 42,9 & \\
\hline Feminino & 104 & 48,6 & 110 & 51,4 & \\
\hline Faixa etária (anos) & & & & & 0,091 \\
\hline $20-29$ & 12 & 36,4 & 21 & 63,6 & \\
\hline $30-39$ & 41 & 45,1 & 50 & 54,9 & \\
\hline $40-49$ & 40 & 57,1 & 30 & 42,9 & \\
\hline 50 e mais & 23 & 60,5 & 15 & 39,5 & \\
\hline Cor da pele & & & & & 0,793 \\
\hline Branca & 28 & 50,9 & 27 & 49,1 & \\
\hline Não branca & 88 & 48,9 & 92 & 51,1 & \\
\hline Estado civil & & & & & 0,751 \\
\hline Com cônjuge & 77 & 50,3 & 76 & 49,7 & \\
\hline Sem cônjuge & 39 & 48,2 & 42 & 51,8 & \\
\hline Escolaridade & & & & & 0,044 \\
\hline Médio completo/Superior incompleto/Técnico & 6 & 46,2 & 7 & 53,8 & \\
\hline Superior completo & 47 & 41,6 & 66 & 58,4 & \\
\hline Especialização/Mestrado/Doutorado & 63 & 58,3 & 45 & 41,7 & \\
\hline Religião & & & & & 0,002 * \\
\hline Agnóstico & 10 & 62,5 & 6 & 37,5 & \\
\hline Católico & 65 & 51,2 & 62 & 48,8 & \\
\hline Kardecista & 14 & 82,3 & 3 & 17,7 & \\
\hline Evangélico (Batista/Adventista) & 6 & 23,1 & 20 & 76,9 & \\
\hline Outras & 21 & 44,7 & 26 & 55,3 & \\
\hline Profissão & & & & & $<0,001$ \\
\hline Enfermeiro & 81 & 44,3 & 102 & 55,7 & \\
\hline Médico/Dentista & 19 & 90,5 & 2 & 9,5 & \\
\hline Outras & 15 & 51,7 & 14 & 48,3 & \\
\hline Função & & & & & $<0,001$ \\
\hline Coordenador/Vice-coordenador & 96 & 57,8 & 70 & 42,2 & \\
\hline Outras & 20 & 29,4 & 48 & 70,6 & \\
\hline Tipo de vínculo com o serviço & & & & & 0,006 * \\
\hline SUS & 114 & 52,3 & 104 & 47,7 & \\
\hline Outros & 2 & 13,3 & 13 & 86,7 & \\
\hline
\end{tabular}

(continua) 
Tabela 1 (continuação)

\begin{tabular}{|c|c|c|c|c|c|}
\hline \multirow[t]{2}{*}{ Variáveis sociodemográficas } & \multicolumn{2}{|c|}{ PIC } & \multicolumn{2}{|c|}{ Não PIC } & \multirow[t]{2}{*}{ Valor de p } \\
\hline & $\mathbf{n}$ & $\%$ & $\mathbf{n}$ & $\%$ & \\
\hline Tempo de trabalho no serviço público (anos) & & & & & 0,001 \\
\hline$\leq 1$ & 5 & 29,4 & 12 & 70,6 & \\
\hline$>1 \leq 5$ & 34 & 49,3 & 35 & 50,7 & \\
\hline$>5 \leq 15$ & 44 & 43,6 & 57 & 56,4 & \\
\hline$>15$ & 30 & 76,9 & 9 & 23,1 & \\
\hline Tempo de trabalho na UBS (anos) & & & & & $<0,001$ \\
\hline$\leq 1$ & 32 & 37,2 & 54 & 62,8 & \\
\hline$>1 \leq 5$ & 50 & 59,5 & 34 & 40,5 & \\
\hline$>5 \leq 15$ & 13 & 38,2 & 21 & 61,8 & \\
\hline$>15$ & 16 & 80,0 & 4 & 20,0 & \\
\hline
\end{tabular}

SUS: Sistema Único de Saúde.

* Teste de Fisher.

Fonte: elaboração própria, com base nos dados da pesquisa A Oferta de Racionalidades Médicas e Práticas Integrativas e

Complementares na Atenção Primária à Saúde da Região Metropolitana de Campinas.

Conclui-se que os gestores dos serviços de ESF no SUS não apenas têm baixo reconhecimento da oferta das PIC, mas atuam para a baixa institucionalização, fragilidade e a instabilidade das PIC nos serviços, além de produzirem, com o seu desapoio, em concordância com os trabalhos de Oliveira 12 e Barros et al. 8,13, humilhação social dos trabalhadores de saúde e invisibilidade pública de suas ações de cuidado assentadas em outras racionalidades médicas diferentes da biomédica.

Nesse cenário, em que tendências políticas e econômicas de segmentação e mercantilização na saúde minam o caráter solidário do SUS 14, o suporte dos diferentes níveis de gestão para a ampliação das PIC na ESF pode potencializar o cuidado da população, bem como revitalizar espaços políticos democráticos e movimentos coletivos de diálogo, participação, autonomia e cidadania 15 .

\section{Colaboradores}

Todos os autores elaboraram conjuntamente o texto.

\section{Informações adicionais}

ORCID: Nelson Filice de Barros (0000-0002-54041516); Priscila Maria Stolses Bergamo Francisco (0000-0001-7361-9961); Leandra Andréia de Sousa (0000-0002-7147-935X).
1. Barbosa FES, Guimarães MBL, Santos CR, Bezerra AFB, Tesser CD, Sousa IMC. Oferta de Práticas Integrativas e Complementares em Saúde na Estratégia Saúde da Família no Brasil. Cad Saúde Pública 2020; 36:e00208818.

2. Contatore OA, Barros NF, Durval MR, Barrio PCCC, Coutinho BD, Santos JA, et al. Uso, cuidado e política das práticas integrativas e complementares na atenção primária à saúde. Ciênc Saúde Colet 2015; 20:3263-73.

3. Sá RF, Nogueira J, Guerra VA. Traditional and complementary medicine as health promotion technology in Brazil. Health Promot Int 2019; 34 Suppl 1:i74-81.

4. Sousa LA, Barros NF, Pigari JO, Braghetto GT, Karpiuck LB, Pereira MJB. Acupuntura no Sistema Único de Saúde - uma análise nos diferentes instrumentos de gestão. Ciênc Saúde Colet 2017; 22:301-10. 
5. Sheikh K, Nambiar D. Government policies for traditional, complementary and alternative medical services in India: from assimilation to integration? Natl Med J India 2011; 24:245-6.

6. Wiesener S, Salamonsen A, Fønnebø V. Which risk understandings can be derived from the current disharmonized regulation of complementary and alternative medicine in Europe? BMC Complement Altern Med 2018; 18:11.

7. Sousa IMC, Tesser CD. Medicina Tradicional e Complementar no Brasil: inserção no Sistema Único de Saúde e integração com a atenção primária. Cad Saúde Pública 2017; 33:e00150215.

8. Barros NF, Spadacio C, Costa MV. Trabalho interprofissional e as Práticas Integrativas e Complementares no contexto da atenção primária à saúde: potenciais e desafios. Saúde Debate 2018; 42:163-73.

9. Galhardi WMP, Barros NF, Leite-Mor ACMB. O conhecimento de gestores municipais de saúde sobre a Política Nacional de Prática Integrativa e Complementar e sua influência para a oferta de homeopatia no Sistema Único de Saúde local. Ciênc Saúde Colet 2013; 18:21320.
10. Galhardi WMP, Barros NF, Leite-Mor ACMB. A homeopatia na rede pública do Estado de São Paulo: facilitadores e dificultadores. Rev Bras Med Fam Comunidade 2012; 7:35-43.

11. Departamento de Atenção Básica, Secretaria de Atenção à Saúde, Ministério da Saúde. Política Nacional de Práticas Integrativas e Complementares no SUS: atitude de ampliação de acesso. 2a Ed. Brasília: Ministério da Saúde; 2015.

12. Oliveira MCS. As (in)visibilidades do lian gong na atenção primária em saúde [Tese de Doutorado]. Campinas: Universidade Estadual de Caminas; 2018.

13. Barros LCN, Oliveira ESF, Hallais JAS, Teixeira RAG, Barros NF. Práticas Integrativas e Complementares na atenção primária à saúde: percepções dos gestores dos serviços. Esc Anna Nery Rev Enferm 2020; 24:e20190081.

14. Teixeira CFS, Paim JS. A crise mundial de 2008 e o golpe do capital na política de saúde no Brasil. Saúde Debate 2018; 42:11-21.

15. Barros NF. Abram alas para os novos movimentos sociais: Saúde da Família e Práticas Integrativas e Complementares. Rev Bras Saúde Fam 2008; 9 (n.spe):62-3.

Recebido em 30/Mar/2020

Aprovado em 02/Abr/2020 